\title{
Authors' response to Ghatak and Gulati
}

\author{
Rajesh Puri ${ }^{1}$
}

Published online: 28 September 2016

(C) Indian Society of Gastroenterology 2016

\section{Dear Sir,}

We are thankful for the authors for the interest in our study and to raise some questions for the sake of clarity. The study was conducted at one hospital only; the authors who contributed significantly as per international guidelines are at different centres. Randomization was done by opening of sealed envelopes as described in the study. At present, there are several studies that have shown that EUS before ERCP precludes the latter in significant proportion of patients with suspected choledocholithiasis; however, at the time of conducting the study, there was limited data available and thus we planned this study. Still at many places, patients are subjected to ERCP directly without doing EUS or MRCP, the study was conducted to give a clear message to society that we should not do ERCP directly in patients with moderate and indeterminate risk of choledocholithiasis. It is also noteworthy that negative predictive value of EUS is not $100 \%$; thus, EUS may miss some patients with choledocholithiasis $[1,2]$. EUS was done in a follow up at 3 and 6 months rather than transcutaneous ultrasound or MRCP as it has better sensitivity for CBD stones, and baseline negative EUS does not provide $100 \%$ negative predictive value. Transcutaneous ultrasound not showing stone in any of the patients is a mere coincidence, and it should be noted that patients with high risk of choledocholithiasis were not included in the study.

\section{References}

1. Petrov MS, Savides TJ. Systematic review of endoscopic ultrasonography versus endoscopic retrograde cholangiopancreatography for suspected choledocholithiasis. Br J Surg. 2009;96:967-74.

2. Kohut M, Nowakowska-Dulawa E, Marek T, Kaczor R, Nowak A. Accuracy of linear endoscopic ultrasonography in the evaluation of patients with suspected common bile duct stones. Endoscopy. 2002;34:299-303.
Rajesh Puri

purirajesh69@gmail.com

1 Institute of Digestive and Hepatobiliary Sciences, Medanta, The Medicity, Gurgaon 122 001, India 